\title{
Vulnerabilidade de mulheres em união heterossexual estável à infecção pelo HIV/Aids: estudo de representações sociais*
}

\author{
VULNERABILITY OF WOMEN IN COMMON-LAW MARRIAGE TO BECOMING INFECTED \\ WITH HIVIAIDS: A STUDY OF SOCIAL REPRESENTATIONS
}

\author{
VULNERABILIDAD DE MUJERES EN UNIÓN HETEROSEXUAL ESTABLE A LA \\ INFECCIÓN POR VIH/SIDA: ESTUDIO DE REPRESENTACIONES SOCIALES
}

\section{Larissa Silva Abreu Rodrigues ${ }^{1}$, Mirian Santos Paiva ${ }^{2}$, Jeane Freitas de Oliveira ${ }^{3}$, Sheva Maia da Nóbrega ${ }^{4}$}

\section{RESUMO}

$\mathrm{O}$ artigo discute as representações sociais de mulheres em união heterossexual estável no que diz respeito à vulnerabilidade à infecção pelo HIV/AIDS. Os dados foram produzidos pela associação livre de palavras e constituem recorte de uma pesquisa fundamentada na Teoria das Representações Sociais desenvolvida com mulheres soronegativas para o HIV, da capital e interior da Bahia. A análise fatorial de correspondência revelou significância para as variáveis: procedência, escolaridade e tempo de união estável. A aceitação à traição emergiu como fator de vulnerabilidade para respondentes com 1-5 anos de união estável do interior. Mulheres da capital com 6-10 anos de união estável representam a monogamia como forma de prevenção. Mulheres com maior tempo de união e nível escolar básico representam-se como invulneráveis, contrárias as que têm 1-5 anos de união e escolaridade mediana. Os resultados indicam a necessidade de mais ações com o objetivo de desnaturalizar as coerções sócio-culturais que geram representações e aproximam mulheres em união estável da AIDS.

\section{DESCRITORES}

Mulheres

Síndrome de Imunodeficiência Adquirida

Gênero e saúde

Vulnerabilidade em saúde

\section{ABSTRACT}

This article discusses the social representations of women living in common-law marriage in terms of their vulnerability to becoming infected with HIV/AIDS. Data were obtained through the free association of words, and consisted of an excerpt of a study founded on the Social Representations Theory developed with HIV-negative women living in the state capital and cities in the interior of Bahia. The correspondence factor analysis showed significance for the variables: origin, education level and time spent in commonlaw marriage. Their acceptance of marital affairs emerged as a vulnerability factor for respondents with one to five years spent in common-law marriage, living in cities in the interior. Women from the capital, with 6-10 years spent in common-law marriage, reported monogamy as a form of prevention. Women with a longer common-law marriage who had completed only a primary education reported feeling invulnerable, which was the opposite of those with one to five years in common-law marriage with a secondary education. Results show there is a need for more interventions aiming to denaturalize the socio-cultural coercions that generate representations and make women in common-law marriages more vulnerable to AIDS.

\section{DESCRIPTORS}

Women

Acquired Immunodeficiency Syndrome

Gender and health

Health vulnerability

\section{RESUMEN}

Se discuten representaciones sociales de mujeres en unión heterosexual estable, respecto de vulnerabilidad a infección por $\mathrm{VIH} / \mathrm{SIDA}$. Datos generados a partir de asociación libre de palabras, constituyéndose en muestra de investigación fundamentada en Teoría de las Representaciones Sociales, desarrollada con mujeres seronegativas para VIH, de capital e interior de Bahía. El análisis factorial de correspondencia reveló significatividad para: procedencia, escolaridad y tiempo de unión. La aceptación de la traición surgió como factor de vulnerabilidad para participantes con 1-5 años de unión estable del interior. Las mujeres de capital con 6-10 años de unión representan a la monogamia como forma de prevención. Mujeres con mayor tiempo de unión y escolaridad básica se auto-representan como invulnerables, al contrario de aquellas con 1-5 años de unión y escolarización media. Los resultados sugieren aumentar acciones para desnaturalizar coerciones socioculturales que engendran representaciones y favorecen la exposición de la mujer en unión estable al SIDA.

\author{
DESCRIPTORES \\ Mujeres \\ Síndrome de Inmunodeficiencia Adquirida \\ Género y salud \\ Vulnerabilidad en salud
}

\footnotetext{
* Extraído da dissertação "Representações de mulheres em união heterossexual estável sobre vulnerabilidade à infecção pelo HIV/Aids, Universidade Federal da Bahia, 2009. ${ }^{1}$ Enfermeira. Mestre em Enfermagem pela Universidade Federal da Bahia. Especialista em Saúde Pública pelo Instituto Superior Ciências do Trabalho e da Empresa. Jequié, BA, Brasil. larissagbi@hotmail.com ${ }^{2}$ Doutora em Enfermagem pela Universidade de São Paulo. Pós-Doutora em Psicologia pelo Instituto Superior Ciências do Trabalho e da Empresa. Professora Associada da Universidade Federal da Bahia. Salvador, BA, Brasil. mirian@ufba.br ${ }^{3}$ Professora Doutora da Universidade Federal da Bahia e do Programa de Pós-Graduação em Enfermagem. Membro do Grupo de Estudos sobre Sexualidades, Vulnerabilidades e Gênero da Escola de Enfermagem da Universidade Federal da Bahia. Salvador, BA, Brasil. jeanefo@ufba.br ${ }^{4}$ Mestre em Psicologia Social pela Universidade Federal da Paraíba. Doutora em Psicologia Social pela Ecole des Hautes Etudes en Sciences Sociales. Diplôme d'Études Aproffondies pela Ecole des Hautes Etudes en Sciences Sociales. Professora da Universidade Federal de Pernambuco. Recife, PE, Brasil. shevamaia@hotmail.com
} 


\section{INTRODUÇÃO}

A susceptibilidade das pessoas à AIDS perpassa contextos de vulnerabilidade e questões de gênero que ao longo dos anos demarcou mudanças no padrão epidemiológico incluindo grupos, inicialmente não considerados como susceptíveis, a exemplos das mulheres em união heterossexual estável/casadas.

O conceito de vulnerabilidade, oriundo da área dos Direitos Humanos, adentra o campo da saúde em 1992, considera que a exposição das pessoas ao adoecimento é resultante da interação de aspectos que envolvem três dimensões as quais estão intimamente interligadas: individuais, programáticas e sociais $^{(1)}$. O caráter multidisciplinar da vulnerabilidade inclui a detecção simultânea de fragilidades e da capacidade de enfrentamento dos problemas/agravos de saúde possibilitando inovações na atenção à saúde e permitindo atendimento às necessidades de saúde para além das questões de ordem física, clínica e biológica ${ }^{(2)}$. Logo, a incorporação do conceito de vulnerabilidade pode contribuir para renovação das práticas de enfermagem ${ }^{(3)}$ na busca de atender as necessidades individuais e/ou de grupos específicos.

A AIDS, compreendida sob o prisma da vulnerabilidade, desnuda nuances e contradições que delineiam as relações de gênero e de poder entre homens e mulheres em contextos distintos. A concepção de gênero como um sistema de signos e símbolos que denota relações de poder e hierarquia entre os sexos e no interior de relações entre pessoas do mesmo sexo ${ }^{(4)}$ possibilita o reconhecimento do impacto das relações de poder sobre a saúde de distintos grupos sociais, com redirecionamento e priorização dos cuidados no cotidiano de unidades de saúde ${ }^{(5)}$.

Construções sociais e culturais acerca da masculinidade e feminilidade, numa transversalidade com classe social, raça, diferenças de geração e cultura estabelecem diferenças de vulnerabilidade para indivíduos e/ou grupos sócias vivendo em uma mesma sociedade ou em sociedades distintas. A heterossexualização e a feminização da epidemia estão relacionados, sobretudo, às estruturas de pensamentos, concepções e representações que determinam, entre outras ações, o não uso dos preservativos nas relações sexuais no contexto do casamento(6).

A representação social da Aids como doença do outro determina distanciamento de pessoas e/ou grupos populacionais para condutas que possibilitam à prevenção da doença, dentre elas a prática de sexo seguro. A associação da AIDS ao enfoque de grupo de risco, influenciada por campanhas educativas que enfatizam os comportamentos sexuais na susceptibilidade ao HIV, determina que as pessoas, de forma geral, se definam como isentas da possibilidade de adquirir a doença, comprometendo, consequentemente, a adoção de ações de prevenção(7).
Estudos sobre a percepção de mulheres casadas/em união estável sobre ações preventivas frente ao HIV/Aids são escassos, sendo ressaltada a interface de significação da infeç̧ão e adoecimento pelo HIV com moralidades relacionadas ao casamento e papéis assumidos pelos parceiros ${ }^{(8)}$. Estudo realizado com mulheres que viviam em união estável a confiança no parceiro representou uma explicação frequente para a não utilização do preservativo constituindo fator de vulnerabilidade para ambos ${ }^{(9)}$. Situação similar foi encontrada no estudo realizado com mulheres moradoras de uma favela da cidade de São Paulo, no Brasil(10).

Considerar o tempo do relacionamento conjugal como seguro ou não constitui uma forma de reconhecer os acontecimentos do cotidiano a partir de explicações oriundas de crenças positivas quanto ao status social do casamento condicionando comportamentos, tanto masculinos quanto femininos, de não prevenção(11).

Em face ao exposto, desenvolveu-se uma pesquisa com objetivo de apreender as representações sociais de mulheres em união heterossexual estável, vivendo em contextos sociais distintos, a respeito da vulnerabilidade à infecção pelo HIV/Aids. A pesquisa foi formulada com o pressuposto que as representações sociais influenciam as práticas e atitudes das pessoas nos diferentes contextos sociais, expondo-as ou não a situações de vulnerabilidade para determinados agravos.

\section{METÓDO}

Os dados apresentados constituem recorte de uma pesquisa do tipo descritiva e exploratória de abordagem quanti-qualitativa, realizado junto a mulheres casadas soronegativas ou sorodesconhecidas, ou seja, que não conhecem sua condição sorológica para o HIV, uma vez que, não realizaram teste laboratorial diagnóstico para o HIV e que moram com o parceiro atual por mais de um ano.

O desenvolvimento do estudo obedeceu aos preceitos éticos dispostos na Resolução 196/96 do Conselho Nacional de Saúde (CNS). O projeto foi aprovado pelos Comitês de Ética em Pesquisa da Escola de Enfermagem da Universidade Federal da Bahia (Parecer no 48/2008) e da Universidade Estadual do Sudoeste da Bahia (Parecer no 217/2008).

O contato com as participantes ocorreu a partir da inserção da pesquisadora, no serviço de planejamento familiar, de duas unidades básicas de saúde localizadas em municípios distintos do estado da Bahia: Salvador, a capital do estado e, Jequié, situado na região centro-sul, distante $365 \mathrm{~km}$ da capital. Salvador detém 51\% do total de casos notificados de AIDS no estado, enquanto em Jequié foram registrados junto ao Centro de Referência em Saúde Sexual e Reprodutiva 223 casos, de 2002 até outubro de 2009. 
No período de fevereiro a maio de 2009, em ambos os municípios, após consulta com a enfermeira, as mulheres foram convidadas a integrar a pesquisa. Depois dos esclarecimentos devidos sobre a pesquisa foi oferecido às participantes o Termo de Consentimento Livre e Esclarecido (TCLE) para leitura e assinatura, conforme preconizado pela Resolução 196/96 do CNS. Entre as abordadas, 195 aceitaram participar da pesquisa respondendo ao teste de associação livre de palavras (TALP), sendo 100 moradoras da capital e 95 do município do interior do estado.

A apreensão das representações sociais das participantes acerca da vulnerabilidade para infecção pelo HIV/Aids se deu pela aplicação de um conjunto de técnicas adequadas ao estudo qualitativo fundamentado na Teoria das Representações Sociais, entretanto, neste artigo serão abordados os dados coletados pela técnica de associação livre de palavras. Trata-se de uma técnica projetiva adaptada da prática clínica para psicologia social que permite trazer a consciência elementos inconscientes por meio de manifestações de condutas de reações, evocações, ou seja, a estrutura da personalidade do sujeito ${ }^{(12)}$.

O instrumento utilizado foi composto de dados sócio demográficos das participantes e quatro estímulos: aids, homem e aids, mulher e aids e você mesma. A definição desses estímulos se deu pela necessidade de identificar como as mulheres expressam questões referentes à Aids, a vulnerabilidade de homens e mulheres e, ainda, como se posicionam neste contexto. O TALP foi aplicado individualmente, sendo apresentado um estímulo de cada vez e registrado as cinco primeiras palavras evocadas para o mesmo. $O$ tempo médio de aplicação do teste foi de quatro minutos para cada participante.

Os dados emergidos do TALP foram submetidos à análise fatorial de correspondência (AFC) através do software Tri-deux-mots, versão 2.2 , sendo considerada frequência igual ou superior a oito em relação a cada estímulo indutor. O processamento dos dados exige organização específica que proporciona correlação entre as variáveis fixas ou sócio-demográficas das informantes (procedência, escolaridade, religião, tempo de casamento) previamente estabelecidas e variáveis de opinião apresentadas pelas muIheres para os estímulos indutores (aids, homem e aids, mulher e aids e você mesma). Para as variáveis fixas foram consideradas as seguintes possibilidades: procedência (capital ou interior), escolaridade (ensino fundamental, ensino médio), religião (evangélica, católica, sem religião) e tempo de casamento ( 1 a 5 anos; 6 a 10 anos; maior ou igual a 11 anos).

O processamento pelo Tri-deux-mots resulta num gráfico que permite visualizar as variáveis de opinião e variáveis fixas com significância estatística e as oposições entre os grupos estudados. 0 gráfico não é auto-explicativo e sua análise exige conhecimentos e habilidades acerca da temática de estudo e da teoria das representações sociais para compreensão dos significados e sentidos das variáveis de opinião evocadas para os estímulos apresentados.

$\mathrm{Na}$ tentativa de facilitar o entendimento dos dados do TALP processados pelo Tri-deux-mots, será apresentada, inicialmente, as variáveis de opinião para cada estímulo numa correlação com as variáveis fixas que apresentaram significância estatística. Em seguida, será apresentada a interpretação dos dados revelados pelo gráfico.

\section{RESULTADOS}

O processamento dos dados pelo software Tri-DeuxMots revelou diferentes conjuntos de evocações para cada estímulo indutor associado à diferente tempo de união estável, procedência e nível de escolaridade das mulheres. A variável fixa religião não apareceu no gráfico o que demonstra que a mesma não apresentou significância estatística entre as demais variáveis.

As evocações que apresentaram significância estatística para o grupo de mulheres com tempo de união estável estimado em 11 anos ou mais, que cursou apenas o ensino fundamental e procedente de para os quatro estímulos foram: incurável, perigosa, parceiro e cuidado para o estímulo 1 (aids); doença transmissível, cuidados, sexo, exames e uso da camisinha para o estímulo 2 (homem e aids); higiene, exames, transmissão da doença e sexo para o estímulo 3 (mulher e aids) e, morte para o estímulo 4 (você mesma).

As mulheres com nível médio de escolaridade e tempo de união estável de 6 a 10 anos, as palavras com significância estatística para cada estímulo foram: estímulo 1 (aids) - transmissível, sexo, desespero e sofrimento; estímulo 2 (homem e aids) - promiscuidade, descuido, desconhecimento, tristeza e amor; estímulo 3 (mulher e aids) - conhecimento, prevenir e morte; estímulo 4 (você mesma) - responsável, saúde, amor e fé em Deus.

Entre as evocações pronunciadas pelo grupo procedente de Salvador, com tempo de união estável entre 6 e 10 anos as que apresentaram significância foram: para o estímulo 1 (aids) - traição, para o estímulo 2 (homem e aids) - triste, descarado, exame, cuidado e camisinha; para o estímulo 3 (mulher e aids) - triste, irresponsabilidade e promiscuidade e, para o estímulo 4 (você mesma) - exame e parceiro fixo, saúde e fé em Deus.

Para o grupo de mulheres com tempo de união estável entre 1 a 5 anos, procedente do interior, os conjuntos de evocações com significância estatística para cada estímulo foram: estímulo 1 (aids) - preconceito, sofrimento, preocupação, perigo, descuido, prevenção; estímulo 2 (homem e a aids) - medo, traição, parceiro, descuido; estímulo 3 (mulher e aids) - conhecimento e medo e para o estímulo 4 (você mesma) apenas o termo medo. 


\section{DISCUSSÃO}

Implicações do tempo de união heterossexual estável e o nível de escolaridade nas representações sociais de mulheres sobre a vulnerabilidade ao HIV/Aids.

A interpretação das evocações expressadas pelo grupo formado por mulheres com maior tempo de casamento e menor escolaridade, independente da procedência, conduz a idéia de reconhecimento do caráter incurável e transmissivel da AIDS e a necessidade de adotar medidas de cuidado para sua prevenção. A construção dessa idéia pode está ancorada em informações divulgadas pela mídia e por profissionais de saúde que, de um modo geral, têm enfatizado o cuidado, sobretudo o uso de camisinha como uma estratégia importante para evitar a Aids.

As medidas de cuidados expressadas - realização de exames/higiene e uso de camisinha - são apresentadas como ações a serem adotadas de forma diferenciada por mulheres e homens. As variáveis de opinião evocadas reproduzem construções sociais e culturais que estabelecem papeis e funções diferenciados para homens e mulheres de tal forma que os cuidados com a higiene e a realização de exames periódicos relacionados com a saúde sexual e a prevenção de doenças são atribuídas como responsabilidade das mulheres, enquanto, o uso da camisinha é uma ação eminentemente masculina. Essas representações revelam questões de gênero traduzidas na compreensão multidimensional e dinâmica do que significa ser homem ou mulher dentro de contextos sociais específicos( ${ }^{(8)}$.

A variável de opinião morte evocada para o estímulo 4 - você mesma - revela o temor das mulheres diante da Aids e o reconhecimento de que trata-se de uma doença que ainda não tem cura e que pode levar á morte. A significância estatística apresentada para o termo morte conduz a idéia de que a Aids não é uma ameaça tão distante para o grupo de mulheres com maior tempo de casamento e menor nível de escolaridade participantes do estudo. A idéia da Aids como doença do outro ${ }^{(6-7)}$ ainda prevalece, contudo, esse outro não se mostra mais tão distante, denotando a necessidade de cuidados permanentes para continuar afastada do risco de contrair a Aids e morrer em decorrência dela.

As variáveis de opinião evocadas por este grupo de mulheres revelam que o maior tempo de união estável e o baixo nível de escolaridade colocam as mulheres em situação de vulnerabilidade para Aids pela predominância de idéias apoiadas em construções sociais e culturais que demarcam subordinação das mulheres aos homens e pela reprodução de informações divulgadas, sobretudo pela mídia, no inicio da epidemia. Os dados assinalam para necessidade de ações educativas que atendam especificidades de grupos populacionais e respeitem diferenças socioculturais dos grupos ${ }^{(13)}$.

As variáveis de opinião evocadas pelo grupo de muIheres com nível de escolaridade médio e tempo de união conjugal entre 5 a 10 anos enfatizam aspectos afetivos que ultrapassam a visão pragmática, biológica e normativa do conhecimento científico sobre a Aids. Reconhecem a possibilidade de transmissão pela via sexual, contudo, esta transmissão está associada à traição do parceiro o que causa situações de desespero e sofrimento. As evocações apresentadas levam a considerar que a traição causa muito mais desespero e sofrimento que a doença em si. A doença é a comprovação da traição e da promiscuidade masculina, da submissão feminina às condutas masculinas social e culturalmente determinadas.

A objetivação da promiscuidade masculina relacionada à Aids está vinculada ao processo de figuração em que comportamentos e modos de vida concretizam a doença e permitem visualizar o indivíduo, dar sentido e explicar o fenômeno social ${ }^{(14)}$. $\mathrm{Na}$ concepção deste grupo, a vulnerabilidade de homens e mulheres à Aids relaciona-se, sobremaneira, com o nível de (des) conhecimento. Quando estimuladas a se posicionarem no contexto da Aids, ancoram sua própria prevenção a partir de atitudes responsáveis, na dimensão da afetividade (do amor na condição de valor cultural) e da religiosidade. Tal fato talvez não seja indicativo de uma representação de sua própria vulnerabilidade ao HIV/Aids, entretanto, representa um avanço, uma vez que, mesmo com pouco envolvimento se questionam no contexto da epidemia.

O grupo com nível médio de escolaridade e com tempo de união estável entre 5 a 10 anos revela-se vulnerável a transmissão, por fazer prevalecer seus afetos de amor (quando pensa em si mesma e no parceiro) no casamento. De acordo com o dicionário de psicologia o amor é definido como

um sentimento de uma pessoa que se dirige a uma outra pessoa precisa e faz a primeira desejar receber da segunda e de lhe dar prazeres sexuais (se trata-se de adultos), ternura, admiração, cooperação, compreensão, proteção ou pelo menos várias destas satisfações ${ }^{(15)}$.

Nessa perspectiva, o amor pressupõe entrega ao outro e, no exercício da sexualidade essa representação pode influenciar o abandono de práticas sexuais seguras, como o uso do preservativo e com que as mulheres, em especial, acreditem estar protegidas ${ }^{(8)}$. 
A reprodução da idéia de que a natureza do sexo masculino requer variedade de parcerias e que o marido tem necessidades que podem ser atendidas fora do ambiente familiar favorece a vulnerabilidade feminina uma vez que a mulher tende a se preocupar menos com essa situação, a fim de evitar transformar uma ação socialmente prevalente em um conflito pessoal(16).

Os diferentes conjuntos de variáveis de opinião apresentados para os estímulos indutores demarcam que o tempo de união estável e o nível de escolaridade assinalam situações de vulnerabilidade diferenciadas para as mulheres em relação à Aids, as quais estão permeadas, sobretudo, pela reprodução de idéias que determinam condutas diferenciadas para homens e mulheres.

Ademais, as evocações das mulheres com menor nível de escolaridade e maior tempo de união estável reproduzem o conhecimento veiculado pelas campanhas publicitárias de prevenção de forma normativa, com pouco envolvimento na questão, ao passo, que as representações de mulheres com maior escolaridade e menor tempo de experiência conjugal são permeadas por questões de ordem afetiva em função da preponderância do amor pelo parceiro.

\section{Implicações da procedência e do tempo de união heterossexual estável nas representações de mulheres sobre a vulnerabilidade ao HIV/Aids}

O grupo procedente da capital e com tempo de experiência conjugal entre 6 e 10 anos representa a vulnerabilidade ao HIV/Aids pela traição. A representação de homem e Aids é relacionada à sexualidade descontrolada do homem. Tal representação demonstra que a maneira como a sexualidade masculina vem sendo construída sustenta a convicção, por parte dessas mulheres, de que os homens são infiéis por natureza e tem a sexualidade expressa de maneira mais intensa que a da mulher ${ }^{(17)}$. Além disso, a sexualidade masculina vista como infectante também se expressa pela relação dos homens com a prostituição(18).

O estímulo mulher e Aids foi representado pelo grupo de Salvador com tempo de união estável entre 6 a 10 anos, a partir da abordagem de grupo de risco. A mulher que tem ou pode ter Aids, para esse grupo, é irresponsável e tem comportamentos promíscuos. Essas mulheres apresentam, pois, uma representação dicotomizada com relação à mulher e à Aids. Por um lado, ela se representa cuidadosa e precavida mantendo relações com parceiro único, atitude diametralmente oposta quando ela pensa na outra mulher, que não ela, assumindo uma atitude acusativa de promiscuidade, irresponsabilidade e de condição degradantemente triste.

A construção das representações pelo grupo de muIheres com tempo de casamento entre 6 a 10 anos procedentes de Salvador, sobre a vulnerabilidade ao HIV/
Aids evidenciou a influência de constructos machistas e patriarcais em que a mulher associa sexo ao casamento como modelo social. Nesse contexto, a mulher relaciona o fato de ser casada e ter parceria fixa como uma forma de proteção contra a Aids ${ }^{(9-19)}$.

Ao contrário do que circula no imaginário social, as mulheres soropositivas para o HIV não apresentam um número de parceiros significativamente diferente com relação às mulheres soronegativas ${ }^{(20)}$.

Ao se representarem na perspectiva de que tem parceria fixa e fé em Deus, o grupo da capital situa-se relativamente distantes da infecção pelo HIV/Aids. De forma não esperada e aparentemente contraditória, o grupo da capital manifesta conservadorismo em suas representações, entretanto, isto pode ser explicado por serem muIheres com maior tempo de casamento, quando comparadas com o grupo que se encontra em oposição.

Por outro lado, o grupo com menor tempo de vida em comum e procedente do interior destaca como aspectos do (con) viver com Aids: o sofrimento e o preconceito. A traição permeia o imaginário social do grupo como possibilidade de determinar maior vulnerabilidade quando há o descuido do parceiro na prevenção. Suas representações desconsideram as vulnerabilidades de gênero, social, programática, que são questões mais amplas que permeiam e ultrapassam tais comportamentos ${ }^{(1)}$.

Esse mesmo grupo representa a mulher e a si própria no contexto da Aids como medo, demonstrando passividade com relação à sua proteção individual, ao passo, que conferem maior importância ao papel social de esposa, cujas práticas sexuais encontram-se submissas ao homem, como função primordial de satisfação das necessidades sexuais do parceiro.

Categorias sociais, como gênero, são vistas como naturalizadas, encontram-se reificadas e, consequentemente, são operativas e resistentes à mudança ${ }^{(14)}$. O contexto social no qual se inserem as desigualdades de gênero, em termos culturais, sociais e econômicos, permeia a construção das representações sociais em um movimento de mão dupla, ao passo que uma determina a outra.

Em suma, as mulheres do interior sentem-se inseguras e assumem posição acusativa do parceiro acerca da traição, revelando o medo do contágio, já que não detêm mecanismos eficazes para sua prevenção efetiva. Em psicologia, medo corresponde a

uma emoção desencadeada por um estímulo que tem o valor de perigo para o organismo que reduz suas defesas gerando condutas de fuga ou de imobilismo(15).

Este grupo, portanto, evidencia uma atitude de submissão ao parceiro, mesmo que seja ele o portador do seu medo em relação à infecção pelo HIV/Aids. 


\section{CONCLUSÃO}

Nos grupos estudados configuraram-se diferenças e semelhanças nas representações sociais sobre a vulnerabilidade à Aids que confirmam a dinâmica das representações sociais e a influencia da procedência, do nível de escolaridade e do tempo de união estável na vulnerabilidade das mulheres. Contudo, as especificidades dos grupos e a metodologia adotada não são suficientes para generalizar os resultados para população feminina vivendo em situações similares.

Os resultados apresentados apontam informações esclarecedoras quanto às diferenças de contextos sócio-culturais nos quais mulheres em união estável vivem evidenciando a influencia da procedência na vulnerabilidade da infecção pelo HIV/Aids.

O jogo de oposições revelado pelo processamento dos dados evidencia conteúdos valorativos de gênero para as mulheres com maior tempo de união conjugal e residente em Salvador, para as quais a representação da Aids está associada à promiscuidade e a irresponsabilidade das mulheres, entre as quais elas não se incluem e se consideram como protegidas pelo fato de ter parceria fixa. Para as mulheres procedentes do interior a representação da Aids está associada à traição pelo parceiro, caracterizada como um comportamento social e culturalmente estabelecido. Curiosamente, os dois grupos minimizam a culpabilidade e responsabilidade masculina com os adjetivos de que são descuidados e o poder do uso da camisinha é exclusivo do homem.

Nas representações sociais apreendidas observam-se a presença de idéias, valores, crenças e construções so-

\section{REFERÊNCIAS}

1. Salleti Filho H, Calazans G, Franca Junior I, Ayres JRC. Vulnerabilidade e prevenção em tempos de AIDS. In: Barbosa RM, Parker R, organizadores. Sexualidades pelo avesso. direitos, identidades e poder. Rio de Janeiro: IMS/UERJ; São Paulo: Ed.34; 1999. p. 49-71.

2. Bertolozzi MR, Nichiata LYI, Takahashi RF, Ciosak SI, Hino P, Val $L F$, et al. The vulnerability and the compliance in Collective Health. Rev Esc Enferm USP [Internet]. 2009 [cited 2010 Mar 15];43(n.esp 2):1326-30. Available from: http://www.scielo. br/pdf/reeusp/v43nspe2/en_a31v43s2.pdf

3. Nichiata LYI, Bertolozzi MR, Takahashi RF, Fracolli LA. The use of the vulnerability concept in the nursing area. Rev Latino Am Enferm. 2008;16(5):923-8.

4. Schiebinger L. O feminismo mudou a ciência? Bauru: EDUSC; 2001. ciais que em determinado momento facilitam e noutros obstaculizam as práticas de prevenção. Dessa forma, torna-se importante através de trabalhos educativos, valorizar ou desencorajar tais representações, considerando os fatores contextuais imbricados.

Ao analisar essas representações, sob uma perspectiva de gênero, salienta-se a necessidade de articular ações intersetoriais não apenas para aumentar a compreensão da vulnerabilidade ao HIV/Aids entre as mulheres casadas, mas, principalmente, para favorecer condições econômicas, sociais e políticas para superar as desigualdades de poder dessas mulheres.

Ademais, ressalta-se a relevância de problematizar e desnaturalizar as desigualdades vivenciadas pelas mulheres em união conjugal estável independente da sua procedência. Neste contexto, salienta-se o papel das (os) profissionais de saúde, em particular, da equipe de enfermagem, na realização de trabalhos educativos junto com as mulheres a partir da disponibilização de informações que contribuam para dar visibilidade aos fatores que aumentam a vulnerabilidade da mulher em união conjugal à Aids.

Salienta-se, contudo, que a problematização de coerções sociais, políticas, econômicas, religiosas e culturais que engendram e aproximam a mulher em união conjugal estável à Aids não deve se restringir à saúde. Merece ser discutida e valorizada também em outros campos, como a educação, a fim de que possibilite mudanças efetivas. Assim, os achados da presente pesquisa podem proporcionar um incremento para implantação e/ou implementação de ações de educação em saúde mais eficazes na prevenção e no combate da Aids.

5. Schraiber LB. Equidade de gênero e saúde: o cotidano das práticas no Programa de Saúde da Família do Recife. In: Vilela W, Monteiro S, organizadores. Gênero e saúde: Programa de Saúde da Família em questão. Rio de Janeiro: ABRASCO; 2005. p. 39-61.

6. Monteiro S. AIDS e proteção: a visão de jovens de um bairro popular. Estudos Feministas [Internet]. 1999 [citado 2010 mar. 12];7(1-2):72-88. Disponível em: http://www.periodicos. ufsc.br/index.php/ref/article/view/11955/11222

7. Thiengo MA, Oliveira DC, Rodrigues BMRD. Representações sociais do HIV/AIDS entre adolescentes: implicações para os cuidados de enfermagem. Rev Esc Enferm USP. 2005;39(1):6876.

8. Maia C, Guilhem D, Freitas D. Vulnerabilidade ao HIV/AIDS de pessoas heterossexuais casadas ou em união estável. Rev Saúde Pública. 2008;42(2):242-8. 
9. Giacomozzi Al, Camargo BV. Eu confio no meu marido: estudo da representação social de mulheres com parceiro fixo sobre prevenção da AIDS. Psicol Teor Prat. 2004; 6(1):31-44.

10. Praça NS, Gualda DMR. Risco de infeç̧ão pelo HIV: como mulheres moradoras em uma favela se percebem na cadeia de transmissão do vírus. Rev Latino Am Enferm. 2003;11(1):14-20.

11. Oltramari LC, Otto LS. Conjugalidade e AIDS: um estudo sobre infecção entre casais. Psicol Soc. 2006;18(3):55-61.

12. Coutinho MPL, Nóbrega SM, Catão MFM. Contribuições teórico-metodológicas acerca dos instrumentos projetivos no campo das representações sociais. In: Coutinho MPL, Oliveira FB, Fortunato $\mathrm{ML}$, organizadores. Representações sociais: abordagem interdisciplinar. João Pessoa: Ed. UFPB; 2003 p. 50-66.

13. Tura LFR. A aids: repensando a prevenção. In: Tura LFR, Moreira ASP. Saúde e representações sociais. João Pessoa: Ed. UFPB; 2005. p. 167-90.

14. Vala J. Representações sociais e psicologia social do conhecimento quotidiano. In: Vala J, Monteiro MBM. Psicologia social. 7ạ ed. Lisboa: Fundação Calouste Gulbenkian; 2006. p. 457-502.
15. Doron R, Parot F. Dictionnaire de psychologie. Paris: PUF; 1991.

16. Kerr-Pontes LRS, González F, Kendall C, Leão EMA, Távora FR, Caminha I, et al. Prevention of HIV infection among migrant population groups in Northeast Brazil. Cad Saúde Pública. 2004;20(1):320-8.

17. Guerriero I, Ayres JRC, Hearst N. Masculinidade e vulnerabilidade ao HIV de homens heterossexuais, São Paulo, SP. Rev Saúde Pública. 2002;36(4):50-60.

18. Gomes R, Nascimento EF. A produção do conhecimento da saúde pública sobre a relação homem-saúde: uma revisão bibliográfica. Cad Saúde Pública. 2006;22(5):901-11.

19. Silva CM, Vargens OMC. Women's perception about female vulnerability to STD and HIV. Rev Esc Enferm USP [Internet]. 2009 [cited 2010 Mar 15];43(2):401-6. Available from: http://www.scielo.br/pdf/reeusp/v43n2/en_a20v43n2.pdf

20. Santos NJS, Barbosa RM, Pinho AA, Villela WV, Aidar T, Filipe EMV. Contextos de vulnerabilidade para o HIV entre mulheres brasileiras. Cad Saúde Pública. 2009;25 Supl 2:S321-33. 